\title{
Wild-type p53-induced phosphatase sensitizes acute myeloid leukemia cells to conventional chemotherapy
}

\section{Vasily Golotin ${ }^{1}$, Ekaterina Belotserkovskaya ${ }^{2}$, Larisa Girshova², Alexey Petukhov ${ }^{2}$, Andrey Zaritsky ${ }^{2}$, and Oleg Demidov ${ }^{1,3}$}

${ }^{1}$ Laboratory of Molecular Medicine, Institute of Cytology of the Russian Academy of Sciences, Tikhoretskiy pr., 4, Saint Petersburg, 194064, Russian Federation

${ }^{2}$ Almazov Federal Medical Research Centre, ul. Akkuratova, 2, 197341,

Saint Petersburg, Russian Federation

3INSERM UMR1231, Laboratory of Excellence LipSTIC and label Ligue Nationale contre le Cancer, 7 Boulevard Jeanne d'Arc, BP 27877, 21078, Dijon Cedex, France

Address correspondence and requests for materials to Oleg Demidov, demidov.on@mail.ru

\section{Abstract}

Recently wild-type p53-induced phosphatase was implicated in the pathogenesis of acute myeloid leukemia (AML) and "pre-leukemia" myeloproliferative conditions. Here we decided to check how the strategy directed to phosphatase inhibition affected sensitivity to conventional chemotherapy. All experiments were conducted on AML cell lines cultivated in vitro. The levels of wild-type p53-induced phosphatase vary in different AML cell lines. The chemical compound GSK2830371 reduced levels of phosphatase and diminished its activity. GSK2830371 did not significantly change the cell cycle distribution of AML cells when used alone or in combination with the anti-cancer chemotherapeutic drug Cytosar but increased caspase-dependent PARP1 cleavage. In contrast with previous studies, we did not observe the negative effect of phosphatase activity inhibition and depletion on cells when a chemical inhibitor was used as monotherapy. Using a combination of GSK2830371 with Cytosar we were able to reduce the threshold of chemotherapy-dependent cytotoxicity and more efficiently eliminate leukemic cells. We propose considering inhibition of wildtype $\mathrm{p53}$-induced phosphatase as a prospective strategy in improving anti-AML therapy.

Keywords: leukemia, AML, chemotherapy, cytarabine

\section{Introduction}

Acute myeloid leukemia (AML) is a frequent hematological malignancy that can develop spontaneously or be induced by chemotherapy or ionizing radiation. It is characterized by the clonal expansion of myeloid progenitors in the bone marrow and peripheral blood. AML has a multigenic nature with many genes involved in the pathogenesis of the disease, mainly genes involved in cell signaling, transcriptional and epigenetic regulation (Cai and Levine, 2019). Many other myeloid proliferative or dysplastic pathologies with time may be transformed into AML. Currently, researchers and physicians count three steps in AML evolution from single mutations in progenitor cells in the bone marrow to fully developed leukemic cells with multiple mutations in various genes.

The first step when the hematopoietic progenitor acquires a mutation that gives it a competitive advantage in more rapid proliferation or resistance to stress is called Clonal Hematopoiesis of Indeterminate Potential, or CHIP. It is characterized by the appearance and expansion of hematopoietic cells with certain mutations detected in at least $1 \%$ of blood cells. It can lead to changes in the functions of the gene and its products, but does not significantly affect the morphology of cells and does not cause any pathological condition in the hemato- 
poietic system immediately, has indeterminate potential in contrast with the clonal detection of cells already associated with morphological changes (Myelodysplastic syndrome, MDS) or disease (Acute myeloid leukemia, AML). CHIP may predispose to the development of MDS, a condition with the appearance of atypical cells in the blood. Myelodysplastic syndrome includes several oncohematological conditions characterized by a defect in the maturation of cells in bone marrow, but usually at the beginning there are no other symptoms except atypical blood formula. In many cases, MDS cells acquire additional mutations and transform into fully malignant leukemic cells, AML.

Wild-type p53-induced phosphatase, WIP1, is one of the major regulators of DNA damage response and the p53 pathway that is frequently compromised in AML by mutations in the TP53 gene or the members of the DNA damage signaling pathway (Uyanik et al., 2017). Despite the recent addition of targeted therapy and the use of epigenetic drugs, the main drug of choice for AML treatment remains cytarabine. Here we investigated the effect of WIP1 inhibition on AML cells' sensitivity to cytarabine treatment.

Recently, Yu et al. showed that without any relation to mutation status in the fifth and sixth exons of the PPM1D gene (WIP1 protein), this gene was overexpressed in bone marrow cells of AML patients in comparison with control bone marrow cells from healthy donors (2020). Other researchers used RNA interference to deplete WIP1 in several AML cell lines. They showed that reducing WIP1 levels with siRNA to protein phosphatase, $\mathrm{Mg}^{2+} / \mathrm{Mn}^{2+}$ dependent $1 \mathrm{D}$ suppressed cell proliferation and promoted cell apoptosis in AML-193 cells and KG-1 cells (Li et al., 2020).

Here we decided to analyze an approach more relevant to clinical practice, by blocking WIP1 activity with a specific chemical inhibitor, GSK2830371. Moreover, we decided to test how WIP1 inhibition affects AML sensitivity to anti-cancer chemotherapy.

\section{Material and methods}

\section{AML cell cultivation}

Cells were obtained from the shared research facility "Vertebrate cell culture collection" supported by the Ministry of Science and Higher Education of the Russian Federation (Agreement \# 075-15-2021-683).

The OCI-AML-2 cell line (DSMZ no.: ACC 99) was cultured using $80-90 \%$ alpha-MEM (with ribooxyribonucleosides and deoxyribonucleosides) $+20 \%$ h.i. FBS. The MONO-MAC-1 cell line (DSMZ ACC 252) was cultured in $90 \%$ RPMI $1640+10 \%$ h.i. FBS +2 mM L-glutamine $+1 \mathrm{x}$ non-essential amino acids $+1 \mathrm{mM}$ sodium pyruvate.

\section{Cell viability assay}

The CyQUANT ${ }^{\text {twx }}$ XTT Cell Viability Assay kit was used to analyze viability. Cells at a concentration of $1,000,000$ per $\mathrm{mL}$ were seeded with the test compounds (GSK2830371 (GSK) (Selleckchem) and Cytosar (Cytarabine) (Astellas Pharma Inc)) in a 96-well plate and cultured for 48 hours at $37^{\circ} \mathrm{C}\left(5 \% \mathrm{CO}_{2}\right)$. The readings were carried out according to the kit's manual.

\section{Western blotting}

A polyacrylamide gel (10\%) was used for protein electrophoresis according to the standard technique. A nitrocellulose membrane (Bio-Rad) with a pore diameter of $0.2 \mu \mathrm{M}$ was used. The transfer of proteins from the gel to the membrane was carried out in a chamber (Bio$\mathrm{Rad})$ at $0.3 \mathrm{~A}$ for 2 hours. After blocking with $5 \%$ milk in PBS-T $(+0.1 \%$ Tween-20), membranes were incubated with specific antibodies (WIP1 F-10 (SantaCruz), PARP (Invitrogen), p-p38 MAPK (Cell Signaling), p-P53 (Ser15) (Cell Signaling)) overnight. After three washes with PBS-T (+ $0.1 \%$ Tween-20), secondary antibodies were applied accordingly and membranes were revealed with ECL reagents.

\section{Flow cytometry}

\section{CELL CYCLE}

The cells were washed in PBS buffer, then fixed by the addition of cold ethanol to a final concentration of $50 \%$ and subsequent incubation for 20 minutes at $-20^{\circ} \mathrm{C}$. Cells were stained at $37^{\circ} \mathrm{C}$ in PBS buffer containing RNase and propidium iodide. Stained cells were then analyzed on a CytoFLEX Beckman Counter in the PE channel.

\section{APOPTOSIS}

All of the procedures were performed according to kit instructions with Dead Cell Apoptosis Kit with Annexin V PE and SYTOX ${ }^{\mathrm{m}}$ Green. Stained cells were then analyzed on a CytoFLEX Beckman Coulter in the APC and FITC channels.

\section{Results}

First, we performed data mining and found that mutation frequency in the PPM1D gene is different in various subsets of AML and AML-related diseases. The data presented in Table 1 indicate that despite a low general frequency of the PPM1D mutations in AML in general, about $1 \%$ of the cases, up to one-fifth of patients with a therapy-related AML or therapy-related pre-AML condition, MDS, with a reported previous history of DNA 
Table 1. PPM1D mutation frequency in CHIP, MDS, AML

\begin{tabular}{l|c|l}
\hline \multicolumn{1}{c|}{ Disease } & $\begin{array}{c}\text { Frequency } \\
\text { of } P \text { PM1D } \\
\text { mutations }\end{array}$ & \multicolumn{1}{c}{ References } \\
\hline Healthy individuals & $0.42 \%$ & Motomura et al., 2019 \\
\hline CHIP & $4.6 \%$ & Genovese et al., 2014 \\
\hline Primary MDS & $3 \%$ & Lindsley et al., 2017 \\
\hline De novo AML & $1.2 \%$ & Kartal-Kaess et al., 2018 \\
\hline Therapy related MDS & $15-20 \%$ & $\begin{array}{l}\text { Hsu et al., 2018; } \\
\text { Lindsley et al., 2017 }\end{array}$ \\
\hline Therapy related AML & $19.5 \%$ & Hsu et al., 2018 \\
\hline
\end{tabular}

damaging agent treatment, had mutations in the regulatory domain of WIP1 phosphatase.

As discussed above, such mutations lead to the expression of a more stable and active enzyme in AML cells that may affect the leukemogenesis and leukemic cells' responsiveness to the therapy. Therefore, we concluded that WIP1 could play a role in the pathogenesis of AML, and the presence of mutations in the PPM1D gene could influence the choice of therapeutic strategy.

Our own Sanger sequencing of the PPM1D gene for more than 20 patient samples from the archives of the Institute of Oncology and Hematology, Almazov National Medical Research Centre, did not reveal any samples with mutations in PPM1D. The absence of mutations may not only indicate the low frequency of mutated PPM1D, but also can point to the necessity of using next generation sequencing with a reading depth of at least 500 copies to detect clones of AML cells with mutations in the PPM1D gene.

In the next step, we decided to analyze WIP1 inhibition effects on AML cells and changes in AML sensitivity to anti-cancer chemotherapy.

For our studies, we selected two AML cell lines that are well-described in the literature: OCI-AML2 and Mono-Mac-1. According to French-British-American classification, they belong to FAB M4 (Acute myelo- monocytic leukemia) and FAB M5 (Acute monocytic leukemia) classes of AML, correspondingly.

First, we analyzed how these two cell lines respond to the basic anti-AML chemotherapeutic drug Cytosar. Figure 1A shows that OCI-AML 2 cells are more sensitive to the first-line treatment drug Cytosar, than Mono-Mac-1 cells (Fig. 1A). Then we tested the toxicity of GSK2830371 (GSK), a highly specific WIP1 inhibitor, on those two AML cell lines in a biologically relevant range of concentrations from $0.1 \mathrm{uM}$ to $5 \mathrm{uM}$. In contrast with previously published studies on the toxicity of WIP1 RNA interference in AML cells (Li et al., 2020), we did not observe the negative effect of WIP1 phosphatase activity inhibition on cell viability when a chemical inhibitor was used as monotherapy. At the same time, the combination of GSK2830371 with Cytosar significantly potentiated the efficiency of chemotherapy in OCI-AML2 cells but not in Mono-Mac-1 cells, which are resistant to Cytosar (Fig. 1B).

As expected, treatment with Cytosar induced intraS-phase checkpoint and delayed cell cycle progression and proliferation of tumor cells (Fig. 1C). Cytarabine activates the major regulator of the intra-S-phase checkpoint, ATR-Chk1 signaling. This signaling was reported as WIP1 phosphatase target previously (Lu et al., 2005; Mesa et al., 2005). GSK2830371 did not change the cell cycle profile either in control cells or in AML cells treated with Cytosar (Fig. 1C).

The caspase-dependent cleavage of PARP1 protein serves as a marker of apoptotic cell death. We observed the maximum intensity of cleaved product $(89 \mathrm{kDa})$ in OCI-AML2 cells treated with a combination of WIP1 inhibitor and chemotherapeutic agent (Fig. 1D). In MonoMac-1 cells, there was no obvious difference between cells treated only with Cytosar or with a combination of Cytosar and GSK2830371, but both samples had an extra band of $92 \mathrm{kDa}$ that was not present in the control and in cells treated only with GSK.

Another marker of apoptosis, the binding of Annexin $\mathrm{V}$ to the outer leaflet of the plasma membrane, was analyzed by flow cytometry. The results showed that,

Fig. 1. WIP1 inhibition sensitizes AML cells to chemotherapy

A - Influence of Cytosar (Cytarabine) on the viability of OCI-AML-2 and MONO-MAC-1 Cell lines.

Cell viability XTT assay was performed after $48 \mathrm{~h}$ of cultivation. The exponential concentration curve was built with Origin PRO (2019) software.

B - The effect of GSK2830371 (GSK) WIP1 inhibitor and chemotherapeutic drug Cytosar on AML cell viability.

Two AML cell lines, OCI-AML-2 and MONO-MAC-1, were cultivated in the presence of GSK2830371 (GSK) and Cytosar for 48h before XTT assay.

-- no drug added; + -1 uM each of the compounds; ++ $-2 \mathrm{uM}$ each of the compounds; ${ }_{-}-p<0.05(n=3)$.

C - The effect of GSK2830371 (GSK) and Cytosar on cell cycle

The cell cycle distribution of treated and non-treated OCI-AML-2 cells were analyzed using PI staining and FACS

D - PARP cleavage assay

The PARP cleavage by caspases was analyzed by Western blot with anti- PARP antibodies (Invitrogen).

E - The effect of GSK2830371 (GSK) and Cytosar on apoptosis

Flow cytometry dot plots for annexin $\mathrm{V}$ and CytotoxGreen staining of the two cell lines after $48 \mathrm{~h}$ of incubation with drugs

$\mathrm{F}$ - WIP1 signaling in AML cells after inhibition

Cells were treated with GSK2830371 (GSK) and Western blot was performed for WIP1, p-p38 MAPK and p-p53, and actin. 
A

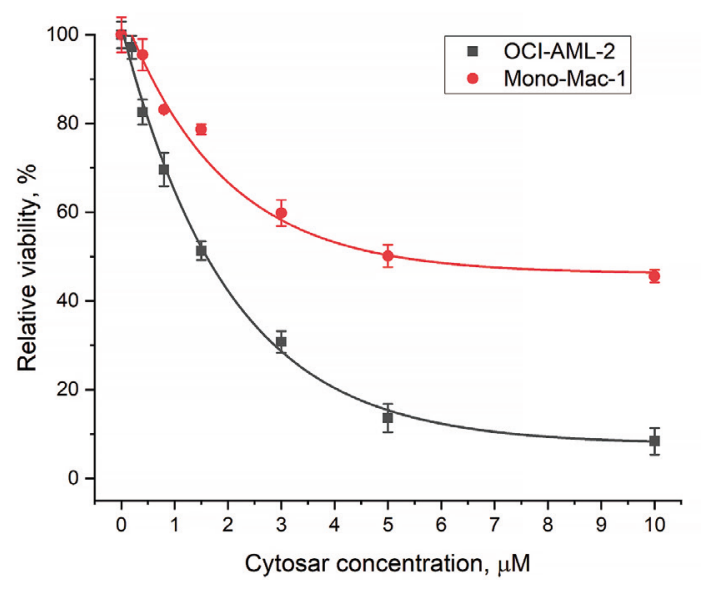

C
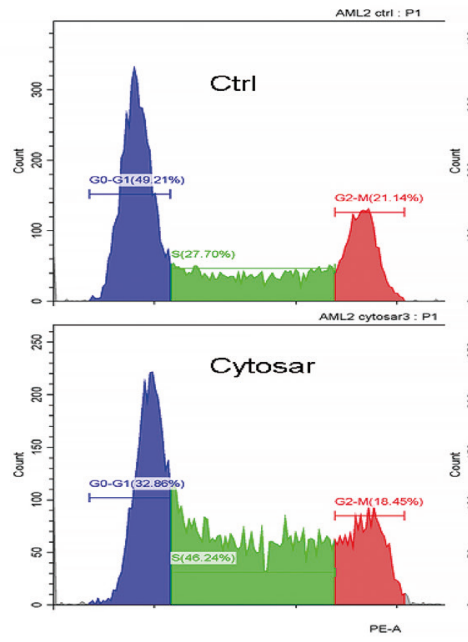

E
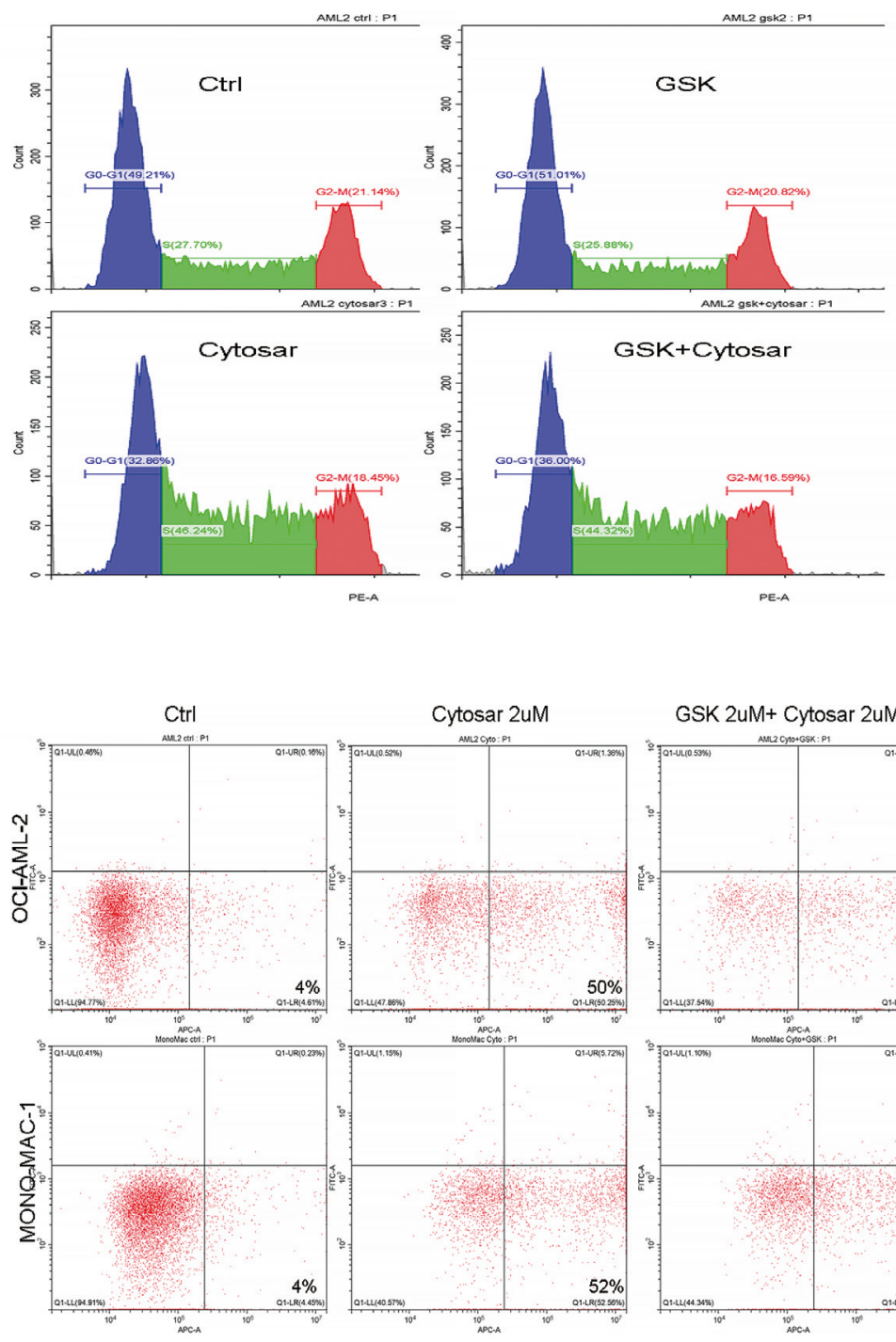

GSK 2UM+ Cytosar 2uM

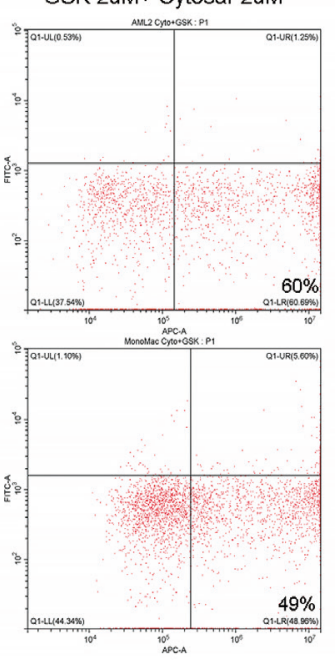

D

\section{OCI-AML-2 M MONO-MAC-1} dmso gsk cyto $g+c \quad c$ trl gsk cyto $g+c$

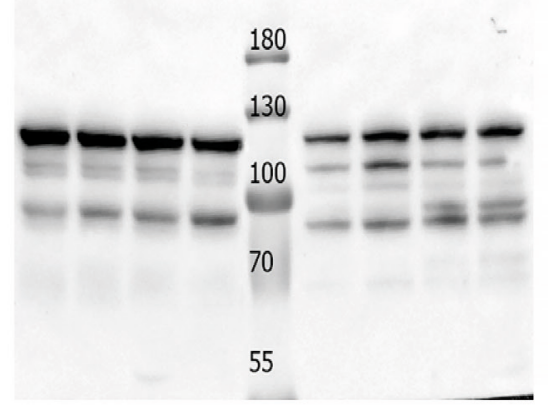

F

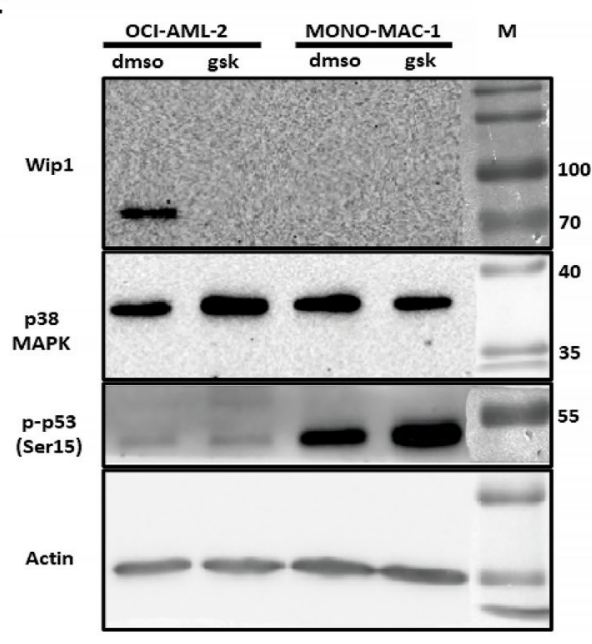


similar to the viability assay, WIP1 inhibitor potentiated efficacy of Cytosar and increased the number of apoptotic cells in treated OCI-AML2 cells. The same concentration of Cytosar induced cell death in Mono-Mac-1 cells to a lesser extent, and there was no difference between the treatment with Cytosar alone and the treatment with a combination of Cytosar and GSK2830371 (Fig. 1E).

Finally, we checked WIP1 downstream signaling and verified the levels of WIP1 in both cell lines. We found that OCI-AML2 had high levels of WIP1 protein. In contrast, we were not able to detect the expression of the PPM1D gene in Mono-Mac1 AML cells with lowaffinity anti-WIP1 SCBT antibodies. As expected from the previous reports, GSK2830371 affected not only phosphatase activity but also reduced protein levels of WIP1 in OCI-AML2 cells (Fig. 1F). One of the main WIP1 targets, p38MAPK kinase was hyperphosphorylated on WIP1-targeted threonine 180 when dephosphorylation activity was inhibited by GSK2830371 in OCI-AML2 cells, but not in WIP1-deficient MonoMac-1 AML cells. p53 transcriptional factor is another WIP1 target linked to apoptosis induction was detected at very low levels in OCI-AML2 cells. The levels of phosphorylated p53 at serine-15 were very high in Mono-Mac-1 AML cells but in this cell line, p53 was inactivated by mutations in the DNA binding domain (p.Arg273His).

\section{Discussion}

In our study, monotherapy with WIP1 inhibitor GSK2830371 did not reduce the number of leukemic cells in comparison with the report published earlier this year, in which researchers used a different shRNA approach to deplete WIP1 in AML cells. WIP1 inhibition strategy in the treatment of AML was inefficient as monotherapy, but it shifted AML cells' response to the widely used chemotherapeutic agent Cytosar towards apoptotic cell death. The difference in the response to WIP1 inhibitor treatment between the two AML cell lines we used in our study can be explained by differences in PPM1D expression. The undetectable levels of WIP1 in Mono-Mac-1 can be linked to the inactive mutant p53. In our previous work, we showed that transcriptional factor p53 could bind to the promoter of the PPM1D gene and could activate its transcription (Rossi et al., 2008). The mutation in TP53 in Mono-Mac-1 cells is located in the DNA binding domain and severely compromised p53 functions as a transcriptional regulator. P53 is a major tumor suppressor and a key protein in DNA damage response. P53 activation by a DNAdamaging chemotherapeutic agent turns on apoptotic cell death. Therefore, the higher resistance of MonoMac- 1 cells to Cytosar can also be explained by inactivating TP53 mutations in this cell line.
Increasing the efficiency of current protocols for the treatment of AML is one of the major tasks in oncohematology. We conclude that WIP1 phosphatase could be a new target in the treatment of AML, and its inhibition potentiates the cytarabine efficiency in inducing cell death of AML cells by favoring apoptosis. At the same time, WIP1 levels in AML cells could be a prognostic marker determining the efficiency of the proposed antitumor strategy.

\section{References}

Cai, S. F. and Levine, R. L. 2019. Genetic and epigenetic determinants of AML pathogenesis. Seminars in Hematology 56(2):84-89. https://doi.org/10.1053/j.seminhematol.2018.08.001

Genovese, G., Kähler, A. K., Handsaker, R. E., Lindberg, J., Rose, S. A., Bakhoum, S. F., Chambert, K., Mick, E., Neale, B. M., Fromer, M., Purcell, S. M., Svantesson, O., Landén, M., Höglund, M., Lehmann, S., Gabriel, S. B., Moran, J. L., Lander, E. S., Sullivan, P. F., Sklar, P., Grönberg, H., Hultman, C.M., and McCarroll, S.A. 2014. Clonal hematopoiesis and blood-cancer risk inferred from blood DNA sequence. The New England Journal of Medicine 371(26):2477-2487. https://doi.org/10.1056/ NEJMoa1409405

Hsu, J. I., Dayaram, T., Tovy, A., De Braekeleer, E., Jeong, M., Wang, F., Zhang, J., Heffernan, T. P., Gera, S., Kovacs, J. J., Marszalek, J. R., Bristow, C., Yan, Y., Garcia-Manero, G., Kantarjian, H., Vassiliou, G., Futreal, P.A., Donehower, L. A., Takahashi, K., and Goodell, M. A. 2018. PPM1D mutations drive clonal hematopoiesis in response to cytotoxic chemotherapy. Cell Stem Cell 23(5):700-713. https://doi.org/10.1016/j.stem.2018.10.004

Kartal-Kaess, M., Bochtler, T., Kraft, B., Kirsch, M., Maier, B., Stoelzel, F., Mohr, B., Kramer, M., Rollig, C., Thiede, C., Bornhäuser, M., Ehninger, G., Müller-Tidow, C., and Krämer, A. 2018. PPM1D mutations are rare in de novo and therapy-related acute myeloid leukemia. Blood 132(Suppl. 1):1472. https://doi.org/10.1182/ blood-2018-99-118566

Li, B., Hu, J., He, D., Chen, Q., Liu, S., Zhu, X., and Yu, M. 2020. PPM1D knockdown suppresses cell proliferation, promotes cell apoptosis, and activates p38 MAPK/p53 signaling pathway in acute myeloid leukemia. Technology in Cancer Research and Treatment 19:1533033820942312. https://doi.org/10.1177/1533033820942312

Lindsley, R. C., Saber, W., Mar, B. G., Redd, R., Wang, T., Haagenson, M. D., Grauman, P. V., Hu, Z. H., Spellman, S. R., Lee, S. J., Verneris, M. R., Hsu, K., Fleischhauer, K., Cutler, C., Antin, J.H., Neuberg, D., and Ebert, B. L. 2017. Prognostic mutations in myelodysplastic syndrome after stem-cell transplantation. The New England Journal of Medicine 376:536-547. https://doi.org/10.1056/NEJMoa1611604

Lu, X., Nannenga, B., and Donehower, L. A. 2005. PPM1D dephosphorylates Chk1 and p53 and abrogates cell cycle checkpoints. Genes and Development 19(10):1162-1174. https://doi.org/10.1101/gad.1291305

Mesa, R. A., Loegering, D., Powell, H. L, Flatten, K., Arlander, S. J., Dai, N. T., Heldebrant, M. P., Vroman, B. T., Smith, B. D., Karp, J. E., Eyck, C. J., Erlichman, C., Kaufmann, S. H., and Karnitz, L. M. 2005. Heat shock protein 90 inhibition sensitizes acute myelogenous leukemia cells to cyta- 
rabine. Blood 106(1):318-327. https://doi.org/10.1182/ blood-2004-09-3523

Motomura, M., Inoue, Y., Nagata, Y., Yoshizato, T., Baer, C., Momozawa, Y., Nadarajah, N., Nannya, Y., Yoshida, K., Haferlach, C., Kern, W., Atsuta, Y., lijima-Yamashita, Y., Shiraishi, Y., Onizuka, M., Chiba, K., Tanaka, H., Itonaga, H., Miyazaki, Y., Horibe, K., Sanada, M., Kamatani, Y., Kubo, M., Miyano, S., Haferlach, T., Ogawa, S., and Makishima, H. 2019. PPM1D and DNMT3A mutations in myelodysplasia and clonal hematopoiesis. Blood 134(Suppl.1):1709. https://doi.org/10.1182/blood-2019-122032

Rossi, M., Demidov, O. N., Anderson, C. W., Appella, E., and Mazur, S. J. 2008. Induction of PPM1D following DNA-damaging treatments through a conserved p53 response element coincides with a shift in the use of transcription initiation sites. Nucleic Acids Research 36(22):7168-7180. https://doi.org/10.1093/nar/gkn888

Yu, M., Hu, J., He, D., Chen, Q., Liu, S., Zhu, X., and Li, B. 2020. Potentiality of protein phosphatase $\mathrm{Mg}^{2+}, \mathrm{Mn}^{2+}-$ dependent 1D as a biomarker for predicting prognosis in acute myeloid leukemia patients. Journal of Clinical Laboratory Analysis 34(5):e23171. https://doi.org/10.1002/ jcla.23171

Uyanik, B., Grigorash, B. B., Goloudina, A. R., and Demidov, O.N. 2017. DNA damage-induced phosphatase Wip1 in regulation of hematopoiesis, immune system and inflammation. Cell Death Discovery 3:17018. https:// doi.org/10.1038/cddiscovery.2017.18 